\title{
THE IMAGES OF LIE POLYNOMIALS EVALUATED ON MATRICES.
}

\author{
ALEXEI KANEL-BELOV, SERGEY MALEV, LOUIS ROWEN
}

\begin{abstract}
Kaplansky asked about the possible images of a polynomial $f$ in several noncommuting variables. In this paper we consider the case of $f$ a Lie polynomial. We describe all the possible images of $f$ in $M_{2}(K)$ and provide an example of $f$ whose image is the set of non-nilpotent trace zero matrices, together with 0 . We provide an arithmetic criterion for this case. We also show that the standard polynomial $s_{k}$ is not a Lie polynomial, for $k>2$.
\end{abstract}

\section{INTRODUCTION}

A Lie polynomial is an element of the free Lie algebra in the alphabet $\left\{x_{i}\right.$ : $i \in I\}$, cf. [Ra, p. 8]. In other words, a Lie polynomial is a sum of Lie monomials $\alpha_{j} h_{j}$, where $h_{j}$ is a Lie word, built inductively: each letter $x_{i}$ is a Lie word of degree 1 , and if $h_{j}, h_{k}$ are distinct Lie words of degree $d_{j}$ and $d_{k}$, then $\left[h_{j}, h_{k}\right]$ is a Lie word of degree $d_{j}+d_{k}$. As usual, a Lie polynomial is multilinear if each letter appearing in $f$ appears exactly once in each of its Lie monomials.

This note, which consists of two parts, is the continuation of BeMR1, in which we considered the question, reputedly raised by Kaplansky, of the possible image set $\operatorname{Im} f$ of a polynomial $f$ on the algebra $M_{n}(K)$ of $n \times n$ matrices over an infinite field $K$ of characteristic 0 or prime to $n$. See [BeMR1] for the historical background. Even the case of Lie identities has room for further investigation. In the first part we are interested in images of Lie polynomials on $M_{n}(K)$, viewed as a Lie algebra, and thus denoted as $\operatorname{gl}_{n}(K)$ (or just $\mathrm{gl}_{n}$ if $K$ is understood). Since $[f, g]$ can be interpreted as $f g-g f$ in the free associative algebra, we identify any Lie polynomial with an associative polynomial; hence. In this way, any set that can arise as the image of a Lie polynomial on the Lie algebra $\mathrm{gl}_{n}$ also fits into the framework of the associative theory of $M_{n}(K)$, and our challenge here is to find examples of Lie polynomials that achieve the sets described in BeMR1, BeMR2, BeMR3.

As we shall see, this task is not so easy as it may seem at first glance. We first consider Lie identities, proving that the standard polynomial

$$
s_{k}:=\sum_{\pi \in S_{k}} \operatorname{sgn}(\pi) x_{\pi(1)} \cdots x_{\pi(k)}
$$

is not a Lie polynomial for $k>2$. Then we classify the possible images of Lie polynomials evaluated on $2 \times 2$ matrices and consider the $3 \times 3$ case, based on

We would like to thank Aner Shalev for interesting and fruitful discussions regarding this paper.

This research was partially supported by the Israel Science Foundation (grant no. 1207/12).

The second named author was partially supported by an ERC grant.

The third author would like to thank the University of Virginia for its support during the preparation of this revision. 
BeMR1, where the field $K$ was required to be quadratically closed, and [M], where results were provided over real closed fields, some of them holding more generally over arbitrary fields. A key role is played by $\mathrm{sl}_{n}$, the Lie algebra of $n \times n$ matrices over $K$ having trace 0 .

In the second part we tie Lie polynomials to the word case.

\section{The images of homogeneous Lie polynomials on gl ${ }_{n} \mathrm{AND} \mathrm{sl}_{n}$}

We refine Kaplansky's question to the Lie case, and ask:

Question 1. What is the possible image set $\operatorname{Im} f$ of a Lie polynomial $f$ on $\mathrm{gl}_{n}$ and $\mathrm{sl}_{n}$ ?

Question 2. For which Lie polynomials $f$ of minimal degree do we achieve this image set? For example, what are the Lie identities of smallest degree on $\mathrm{gl}_{n}$ and $\mathrm{sl}_{n}$ ?

Even the case of Lie identities is nontrivial, although it has already been studied in two important books $\mathrm{Bak}, \mathrm{Ra}$. At the outset, the situation for Lie polynomials is subtler than for regular polynomials, for the simple reason that the most prominent polynomials in the theory, the standard polynomial $s_{n}$ and the Capelli polynomial $c_{n}$, turn out not to be Lie polynomials.

In order to pass to the associative theory, we make use of the adjoint algebra $\operatorname{ad} L=\left\{\operatorname{ad}_{a}: L \rightarrow L: a \in L\right\}$ given by $\operatorname{ad}_{a}(b)=[a, b]$. Note that

$$
\operatorname{dim}_{K}(\operatorname{ad} L)<\operatorname{dim}_{K} \operatorname{End}_{K}(L)=\left(\operatorname{dim}_{K} L\right)^{2} .
$$

Also, it is well-known that the map $a \mapsto \operatorname{ad}_{a}$ defines a Lie homomorphism $L \rightarrow \operatorname{ad} L$.

We write $\left[a_{1}, \ldots, a_{t}\right]$ for $\left[a_{1}, \ldots,\left[a_{t-1}, a_{t}\right]\right]$, and $\left[a^{(k)}, a_{t}\right]$ for $\left[a, \ldots, a, a_{t}\right]$ where $a$ occurs $k$ times. By ad-monomial we mean a term $\alpha \operatorname{ad} x_{i_{1}} \cdots \operatorname{ad} x_{i_{t}}$ for some $\alpha \in K$. By ad-polynomial we mean a sum of ad-monomials.

\section{Remark 1.}

$$
\operatorname{ad}_{a_{1}} \cdots \operatorname{ad}_{a_{t}}(a)=\left[a_{1}, \ldots, a_{t}, a\right] .
$$

In this way, any ad-monomial corresponds to a Lie monomial, and thus any adpolynomial $f\left(\operatorname{ad}_{x_{1}}, \ldots, \operatorname{ad}_{x_{t}}\right)$ gives rise to a Lie polynomial $f\left(x_{1}, \ldots, x_{t}, y\right)$ taking on the same values, and in which $y$ appears of degree 1 in each Lie monomial in the innermost set of Lie brackets.

Recall that an associative polynomial $f\left(x_{1}, \ldots, x_{k}\right)$ is alternating in the last $m+1$ variables if $f$ becomes 0 whenever two of the last $m+1$ variables are specialized to the same quantity. This yields:

Proposition 1. Suppose $L$ is a Lie algebra of dimension $m$, and $f\left(x_{1}, \ldots, x_{k}\right)$ is a multilinear polynomial alternating in the last $m+1$ variables. Then

$$
f\left(\operatorname{ad}_{x_{1}}, \ldots, \operatorname{ad}_{x_{k}}\right)(y)
$$

corresponds to a Lie identity of $L$ of degree $\operatorname{deg} f+1$.

Proof. The alternating property implies $f\left(x_{1}, \ldots, x_{k}\right)$ vanishes on $\operatorname{ad} L$, cf. Row, Proposition 1.2.24], so every substitution of $f\left(\operatorname{ad}_{x_{1}}, \ldots, \operatorname{ad}_{x_{k}}\right)(y)$ vanishes.

Since the alternating polynomial of smallest degree is the standard polynomial $s_{m+1}$, we have a Lie identity of degree $m+2$ for any Lie algebra of dimension $m$. In particular, $\operatorname{dim}\left(\mathrm{sl}_{n}\right)=n^{2}-1$, yielding: 
Corollary 1. $\mathrm{sl}_{n}$ satisfies a Lie identity of degree $n^{2}+1$.

Conversely, we have:

Proposition 2. Suppose $f\left(x_{1}, \ldots, x_{t}, y\right)$ is a Lie polynomial in which y appears in degree 1 in each of its Lie monomials. Then $f$ corresponds to an ad-polynomial taking on the same values on $L$ as $f$.

Proof. In view of Remark 1, it suffices to show that any Lie monomial $h$ can be rewritten in the free Lie algebra as a sum of Lie monomials in which $y$ appears (in degree 1) in the innermost set of Lie brackets. This could be done directly by means of the Jacobi identity, but here is a slicker argument.

Write $h=\left[h_{1}, h_{2}\right]$, and we appeal to induction on the degree of $h . y$ appears say in $h_{2}$. If $h_{1}=y$ then we are done since $h=-\left[h_{2}, y\right]$ corresponds to $-\operatorname{ad}_{h_{2}}$. Likewise if $h_{2}=y$. In general, by induction, $h_{1}$ corresponds to some ad-monomial $\operatorname{ad}_{x_{i_{1}}} \cdots \operatorname{ad}_{x_{i_{k}}}(y)$ and $h_{2}$ corresponds to some ad-monomial $\operatorname{ad}_{x_{i_{k+1}}} \cdots \operatorname{ad}_{x_{i_{\ell}}}(y)$, so $\left[h_{1}, h_{2}\right]$ corresponds to $\operatorname{ad}_{x_{i_{1}}} \cdots \operatorname{ad}_{x_{i_{k}}}\left(\left(\operatorname{ad}_{x_{i_{k+1}}} \cdots \operatorname{ad}_{x_{i_{\ell}}}\right)(y)\right)=\operatorname{ad}_{x_{i_{1}}} \cdots \operatorname{ad}_{x_{i_{\ell}}}(y)$ as desired.

Corollary 2. Any homogeneous Lie polynomial of degree $\geq 3$ must be an identity (viewing the Lie commutator $[a, b]$ as $a b-b a$ ) of the Grassmann algebra $G$.

Proof. Each term includes $\left[x_{i}, x_{j}, x_{k}\right]$, which is well known to be an identity of $G$.

Corollary 1 gives rise to the following special case of Question 2:

Question 3. What is the minimal degree $m_{n}$ of a Lie identity of $\mathrm{sl}_{n}$ ?

By Corollary 1, $m_{n} \leq n^{2}+1$, and in particular $m_{2} \leq 5$. Even the answer $m_{2}=5$ given in [Ra, Theorem 36.1], is not easy, although a reasonably fast combinatoric approach is given in $\mathrm{Bak}$, p. 165], where it is observed that any since any Lie algebra $L$ satisfying a Lie identity of degree $<5$ is solvable, one must have $m_{2} \geq 5$, yielding $m_{2}=5$. Špenko [ $[\breve{S}$, Proposition 7.5] looked at this from the other direction and showed that if $p$ is a Lie polynomial of degree $\leq 4$ then $\operatorname{Im} p=\mathrm{sl}_{2}$.

\section{Example 1.}

(i) The standard polynomial $s_{2}$ itself is a Lie polynomial.

(ii) $s_{4}$ vanishes on $\mathrm{sl}_{2}$ (viewed inside the associative algebra $M_{2}(K)$ ), since $\mathrm{sl}_{2}$ has dimension 3. But surprisingly, this is not the polynomial of lowest degree vanishing on $\mathrm{sl}_{2}$, as we see next.

(iii) Bakhturin [Bak, Theorem 5.14] points out that $f=\left[\left(x_{1} x_{2}+x_{2} x_{1}\right), x_{3}\right]$ vanishes on $\mathrm{sl}_{2}$. In other words, $a_{1} a_{2}+a_{2} a_{1}$ is scalar for any $2 \times 2$ matrices $a_{1}, a_{2}$ of trace 0 . Indeed, $a_{i}^{2}$ is scalar for $i=1,2$, implying $a_{1} a_{2}+a_{2} a_{1}$ is scalar unless $a_{1}, a_{2}$ are linearly independent, in which case $a_{1} a_{2}+a_{2} a_{1}$ commutes with both $a_{1}$ and $a_{2}$, and thus again is scalar. But $f$ is not a Lie polynomial, as seen via the next lemma.

This discussion motivates us to ask when a polynomial is a Lie polynomial. Here is a very easy criterion which is of some use.

Lemma 1. Any Lie polynomial which vanishes on $\mathrm{sl}_{n}$ is an identity of $\mathrm{gl}_{n}$.

Proof. Immediate, since $\mathrm{gl}_{n}^{\prime}=\mathrm{sl}_{n}^{\prime}$. 
The standard polynomial $s_{4}$ is not a Lie polynomial. Here are three ways of seeing this basic fact.

(i) Confront Example 1 with the fact that $m_{2}=5$, whereas $\operatorname{deg} s_{2}=4$.

(ii) A computational approach. We have 15 multilinear Lie monomials of degree 4 , namely $\frac{1}{2}\left(\begin{array}{l}4 \\ 2\end{array}\right)=3$ of the form

$$
\left[\left[x_{i_{1}}, x_{i_{2}}\right],\left[x_{i_{3}}, x_{i_{4}}\right]\right]
$$

and $2\left(\begin{array}{l}4 \\ 2\end{array}\right)=12$ of the form

$$
\left[\left[\left[x_{i_{1}}, x_{i_{2}}\right], x_{i_{3}}\right], x_{i_{4}}\right] .
$$

But

$$
\begin{aligned}
& \left.\left[\left[x_{i_{1}}, x_{i_{2}}\right],\left[x_{i_{3}}, x_{i_{4}}\right]\right]=\operatorname{ad}_{\left[x_{i_{3}}, x_{i_{4}}\right.}\right]_{x_{i_{2}}}\left(x_{i_{1}}\right) \\
& \quad=\operatorname{ad}_{x_{i_{3}}} \operatorname{ad}_{x_{i_{4}}} \operatorname{ad}_{x_{i_{2}}}\left(x_{i_{1}}\right)-\operatorname{ad}_{x_{i_{4}}} \operatorname{ad}_{x_{i_{3}}} \operatorname{ad}_{x_{i_{2}}}\left(x_{i_{1}}\right),
\end{aligned}
$$

so we can rewrite the equations (11) in terms of (2). Furthermore, with the help of the Jacobi identity, (2) can be reduced to seven independent Lie monomials, and one can show that these do not span $s_{4}$. Even though this might seem unduly complicated, it provides a general program to verify that a given polynomial is not Lie.

(iii) The third approach is simpler and works for $s_{k}$, for any $k>2$.

P.M. Cohn was the first to tie the standard polynomial to the infinite dimensional Grassmann algebra $G$ with base $e_{1}, e_{2}, \ldots$, by noting that $s_{k}\left(e_{1}, \ldots, e_{k}\right)=k ! e_{1} \cdots e_{k} \neq 0$ when $k ! \neq 0$. Rosset [Ros used $G$ to reprove the Amitsur-Levitzki Theorem, and recent interest has resurged in studying standard identities via $G$, cf. BrPS, [P2, I] and also in the context of Lie algebras DPP.

Theorem 1. The standard polynomial $s_{k}$ is not a Lie polynomial, for any $k>2$.

Proof. Otherwise, by Corollary 2 it would be an identity of $G$, contradicting Cohn's observation (taking Char $K=0$ ).

2.0.1. A strategy for computing Lie identities. Lemma 1 also can be used to determine when a Lie polynomial $f$ is an identity of $\mathrm{sl}_{n}$. Indeed, this holds iff it is a PI of $M_{n}(K)$, and thus of any central simple $K$-algebra, in particular the symbol algebra $(\alpha, \beta)$, given by

$$
\begin{gathered}
a^{n}=\alpha, \quad b^{n}=\beta, \\
a b=\rho b a
\end{gathered}
$$

where $\rho$ is a primitive $n$-root of 1 . This algebra is spanned by the base $\left\{a^{i} b^{j}: 0 \leq\right.$ $i, j<n\}$, and in some ways this is a better test set for a Lie monomial than the matrix units, because

$$
\left[a^{i} b^{j}, a^{k} b^{\ell}\right]=\left(\rho^{j k}-\rho^{i \ell}\right) a^{i+k} b^{j+\ell} .
$$

Writing $\operatorname{ad}_{(i, j)}$ for $\operatorname{ad}_{a^{i} b^{j}}$ we thus have $\operatorname{ad}_{(i, j)}\left(a^{k} b^{\ell}\right)=\left(\rho^{j k}-\rho^{i \ell}\right) a^{i+k} b^{j+\ell}$.

Let us iterate: Given $\left(i_{1}, j_{1}\right), \ldots,\left(i_{m}, j_{m}\right)$ which we denote as $(\mathbf{i}, \mathbf{j})$, we also notate $\operatorname{ad}_{(\mathbf{i}, \mathbf{j})}:=\operatorname{ad}_{\left(i_{m}, j_{m}\right)} \cdots \operatorname{ad}_{\left(i_{1}, j_{1}\right)}$, and $\overline{\mathbf{i}}_{m}=\sum_{u=1}^{m} i^{m}$, and $\mathbf{j}_{m}=\sum_{u=1}^{m} j^{m}$. An easy induction argument yields

$$
\operatorname{ad}_{\left(i_{m}, j_{m}\right)}\left(a^{k} b^{\ell}\right)=\rho^{\prod_{m}\left(j_{m}\left(\bar{j}_{m-1}+k\right)-i_{m}\left(\bar{i}_{m-1}+\ell\right)\right.} a^{\bar{i}_{m}+k} b^{\bar{j}_{m}+\ell} .
$$


Thus, writing a Lie polynomial as

$$
f=\sum \alpha_{(\mathbf{i}, \mathbf{j})} \operatorname{ad}_{(\mathbf{i}, \mathbf{j})}
$$

we need to solve

$$
\sum \rho \prod_{m}\left(j_{m}\left(\bar{j}_{m-1}+k\right)-i_{m}\left(\bar{i}_{m-1}+\ell\right) a^{\bar{i}_{m}+k} b^{\bar{j}_{m}+\ell}=0\right.
$$

taken over $m$ ! possible rearrangements $\left(i_{\pi}(1), j_{\pi}(1)\right), \ldots,\left(i_{\pi}(m), j_{\pi}(m)\right)$ of $(\mathbf{i}, \mathbf{j})$.

In the generic case, we can take $\alpha, \beta$ to be commuting indeterminates. Now writing a Lie polynomial as $\sum_{u} c_{u} h_{u}$ for $c_{u} \in K$ and Lie monomials $h_{u}$, we evaluate all $c_{u} \operatorname{ad}_{\left(i_{1}, j_{1}\right)} \cdots \operatorname{ad}_{\left(i_{m}, j_{m}\right)}$ on all $a^{k} b^{\ell}$, noting that there are $\left(n^{2}\right)^{m+1}$ equations to solve, and we need the minimal $m$ such that the rank of the coefficient matrix is less than the number of variables. The nontrivial solutions provide the Lie identities. This can be done on the computer for any given $n$, although we do not have a result for general $n$.

2.1. The case $n=2$. Recall from Corollary 1 that there is a Lie identity of degree 5 .

Theorem 2. If $f$ is a homogeneous Lie polynomial evaluated on the matrix ring $M_{2}(K)$, where $K$ is an algebraically closed field, then $\operatorname{Im} f$ is either $\{0\}$, or $K$ (the set of scalar matrices), or the set of all non-nilpotent matrices having trace zero, or $\mathrm{Sl}_{2}(K)$, or $M_{2}(K)$.

Remark 2. The case of scalar matrices in Theorem 2 is possible only when Char $K=2$, and the last case $M_{2}(K)$ is possible only if $\operatorname{deg} f=1$.

Proof of Theorem 2 According to BeMR1, Theorem 1] the image of $f$ must be either $\{0\}$, or $K$, or the set of all non-nilpotent matrices having trace zero, or $\mathrm{sl}_{2}(K)$, or a dense subset of $M_{2}(K)$ (with respect to Zariski topology). Note that if at least one matrix having nonzero trace belongs to the image of $f$ then $\operatorname{deg} f=1$ and thus $\operatorname{Im} f=M_{2}(K)$.

Theorem 3. For any algebraically closed field $K$ of characteristic $\neq 2$, the image of any Lie polynomial $f$ (not necessarily homogeneous) evaluated on $\operatorname{sl}_{2}(K)$ is either $\mathrm{sl}_{2}(K)$, or $\{0\}$, or the set of trace zero non-nilpotent matrices.

Proof. For $f$ not a PI, we can write $f=f_{j}+f_{j+1}+\cdots+f_{d}$, where each $f_{i}$ is a homogeneous Lie polynomial of degree $i$, and $f_{d}$ is not PI. Therefore for any $c \in K$ we have

$$
f\left(c x_{1}, c x_{2}, \ldots, c x_{m}\right)=c^{j} f_{j}+\cdots+c^{d} f_{d} .
$$

Since $f_{d}$ is not $P I$, we can take specializations of $x_{1}, \ldots, x_{m}$ for which $\operatorname{det}\left(f_{d}\right) \neq 0$. Fixing these specializations, we consider $\operatorname{det}\left(c^{j} f_{j}+\cdots+c^{d} f_{d}\right)$ as a polynomial in $c$ of degree $j+\cdots+d$. Since the leading coefficient is not zero and $K$ is algebraically closed, its image is $K$. Thus for any $k \in K$ there exist $x_{1}, \ldots, x_{m}$ for which $\operatorname{det}(f)=k$. Hence (for Char $K \neq 2$ ) any matrix with nonzero eigenvalues $\lambda$ and $-\lambda$ belongs to $\operatorname{Im} f$. Therefore $\operatorname{Im} f$ is either $\mathrm{sl}_{2}$ or the set of trace zero non-nilpotent matrices.

Let us give examples of Lie polynomials having such images: 
Example 1. If Char $K=2$, then $\operatorname{Im} f=K$ also is possible: We take

$$
f(x, y, z, t)=[[x, y],[z, t]] .
$$

Any value of $f$ is the Lie product of two trace zero matrices $s_{1}=[x, y]$ and $s_{2}=[z, t]$. Both can be written as $s_{i}=h_{i}+u_{i}+v_{i}$, where the $h_{i}$ are diagonal trace zero matrices (which are scalar since Char $K=2$ ), the $u_{i}$ are proportional to $e_{12}$, and the $v_{i}$ are proportional to $e_{21}$. Thus $\left[s_{1}, s_{2}\right]=\left[u_{1}, v_{2}\right]+\left[u_{2}, v_{1}\right]$ is scalar.

Over an arbitrary field, $\operatorname{Im} f$ can indeed be equal to $\{0\}$, or $K$, or the set of all non-nilpotent matrices having trace zero, or $\mathrm{sl}_{2}(K)$, or $M_{2}(K)$.

(i) $\operatorname{Im} x=M_{2}(K)$.

(ii) $\operatorname{Im}[x, y]=\mathrm{sl}_{2}$.

(iii) Next, we construct a Lie polynomial whose image evaluated on $\operatorname{sl}_{2}(K)$ is the set of all non-nilpotent matrices having trace zero. We take the multilinear polynomial $h\left(u_{1}, \ldots, u_{8}\right)$ constructed in [DK] by Drensky and Kasparian which is central on $3 \times 3$ matrices. Given $2 \times 2$ matrices $x_{1}, \ldots, x_{9}$ we consider the homogeneous Lie polynomial

$$
f\left(x_{1}, \ldots, x_{9}\right)=h\left(\operatorname{ad}_{\left[x_{9}, x_{9}, \ldots, x_{9}, x_{1}\right]}, \operatorname{ad}_{x_{2}}, \operatorname{ad}_{x_{3}}, \ldots, \operatorname{ad}_{x_{8}}\right)\left(x_{9}\right) .
$$

For any $2 \times 2$ matrix $x, \operatorname{ad}_{x}$ is a $3 \times 3$ matrix since $\operatorname{sl}_{2}$ is 3 -dimensional; hence, for any values of $x_{i}$, the value of $f$ has to be proportional to $x_{9}$. However for $x_{9}$ nilpotent, this must be zero, since $\left[x^{(3)}, y\right]=0$ for any $y \in \operatorname{sl}_{2}(K)$ if $x$ is nilpotent. (When we open the brackets we have the sum of 8 terms and each term equals $x^{k} y x^{3-k}$. But for any integer $k$, either $k \geq 2$ or $3-k \geq 2$.) Thus the image of $f$ is exactly the set of non-nilpotent trace zero matrices.

Another example of a homogeneous Lie polynomial with no nilpotent values is $f(x, y)=[[[x, y], x],[[x, y], y]]$. (See [BGKP, Example 4.9] for details.)

2.2. The case $n=3$. New questions arise concerning the possible evaluation of Lie polynomials on $M_{n}(K)$.

According to [BeMR2, Theorem 3], if $p$ is a homogeneous polynomial with trace vanishing image, then $\operatorname{Im} p$ is one of the following:

- $\{0\}$,

- the set of scalar matrices (which can occur only if Char $K=3$ ),

- a dense subset of $\operatorname{sl}_{3}(K)$, or

- the set of 3-scalar matrices, i.e., with eigenvalues $\left(c, c \omega, c \omega^{2}\right)$, where $\omega$ is our cube root of 1 .

Drensky and Rashkova [DR] have found several identities of $\mathrm{sl}_{3}$ of degree 6 , but they cannot be Lie polynomials, since otherwise they would be identities of $\mathrm{gl}_{3}$ and thus a multiple of $s_{6}$, which is not a Lie polynomial. Thus, one must go to higher degree.

In the associative case, the fact that the generic division algebra has a 3-central element implies that there is a homogeneous 3-central polynomial $f$ for $M_{3}(K)$, i.e., all of whose values take on eigenvalues $c, \omega c, c \omega^{2}$, where $\omega$ is a cube root of 1 . But any matrix with these eigenvalues is either scalar or has trace 0 . This leads us to the basic questions needed to complete the case $n=3$ :

Question 4. Is there a Lie polynomial $f$ whose values are dense on $\operatorname{sl}_{3}(\mathbb{C})$ but do not take on all values? 
Question 5. Is there a Lie polynomial $f$ whose values on $\mathrm{sl}_{3}$ all take on eigenvalues $c, \omega c, c \omega^{2}$, where $\omega$ is a primitive cube root of 1 ?

\subsection{A Group theoretical question and its relation to the Lie theoretical problem.}

Let $w$ be an element of the free group of $m$ letters $x_{1}, x_{2}, \ldots x_{m-1}$ and $x_{m}$. Given a group $G$, we consider the map $f_{w, G}: G^{m} \rightarrow G$ corresponding to the word $w$. This map is called a word map, which for convenience we also notate as $w$ instead of $f_{w, G}$. There is a group conjecture (see BeKP, Question 2] for the more general case):

Conjecture 1. If the field $K$ is algebraically closed of characteristic 0 , then the image of any nontrivial group word $w\left(x_{1}, \ldots, x_{m}\right)$ on the projective linear group $\mathrm{PSL}_{2}(K)$ is $\mathrm{PSL}_{2}(K)$.

Remark 3. Note that if one takes the group $\mathrm{SL}_{2}$ instead of $\mathrm{PSL}_{2}$, Conjecture 1 fails, since the matrix $-I+e_{12}$ does not belong to the image of the word map $w=x^{2}$.

Example 2. When Char $K=p>0$, the image of the word map $w(x)=x^{p}$ evaluated on $\mathrm{PSL}_{2}(K)$ is not $\mathrm{PSL}_{2}(K)$. Indeed, otherwise the matrix $I+e_{12}$ could be written as $x^{p}$ for $x \in \mathrm{PSL}_{2}(K)$. If the eigenvalues of $x$ are equal, then $x=I+n$ where $n$ is nilpotent. Therefore $x^{p}=(I+n)^{p}=I+p n=I$. If the eigenvalues of $x$ are not equal, then $x$ is diagonalizable and therefore $x^{p}$ is also diagonalizable, a contradiction.

Lemma 2 (Liebeck, Nikolov, Shalev, cf. also G] and Ban]). Im $w$ contains all matrices from $\mathrm{PSL}_{2}(K)$ which are not unipotent.

Proof. According to $\mathrm{BO}$ the image of the word map $w$ must be Zariski dense in $\mathrm{SL}_{2}(K)$. Therefore the image of $\operatorname{tr} w$ must be Zariski dense in $K$. Note that $\operatorname{tr} w$ is a homogeneous rational function and $K$ is algebraically closed. Hence, $\operatorname{Im}(\operatorname{tr} w)=K$. For any $\lambda \neq \pm 1$ any matrix with eigenvalues $\lambda$ and $\lambda^{-1}$ belongs to the image of $w$ since there is a matrix with trace $\lambda+\lambda^{-1}$ in $\operatorname{Im} w$ and any two matrices from $\mathrm{SL}_{2}$ with equal trace (except trace \pm 2 ) are similar. Note that the identity matrix $I$ belongs to the image of any word map.

However the question whether one of the matrices $\left(I+e_{12}\right)$ or $\left(-I-e_{12}\right)$ (which are equal in $\mathrm{PSL}_{2}$ ) belongs to the image of $w$ remains open. We conjecture that $I+e_{12}$ must belong to $\operatorname{Im} w$. Note that if there exists $i$ such that the degree of $x_{i}$ in $w$ is $k \neq 0$ then we can consider all $x_{j}=I$ for $j \neq i$ and $x_{i}=I+e_{12}$. Then the value of $w$ is $\left(I+e_{12}\right)^{k}=I+k e_{12}$ and this is a unipotent matrix since Char $K=0$, and thus $\operatorname{Im} w=\operatorname{PSL}_{2}(K)$. Therefore it is interesting to consider word maps $w\left(x_{1}, \ldots, x_{m}\right)$ such that the degree of each $x_{i}$ is zero.

This is why Conjecture 1 can be reformulated as follows:

Conjecture 2. Let $w\left(x_{1}, \ldots, x_{m}\right)$ be a group word whose degree at each $x_{i}$ is 0 . Then the image of $w$ on $G=\mathrm{GL}_{2}(K) /\{ \pm 1\}$ must be $\mathrm{PSL}_{2}(K)$.

One can consider matrices $z_{i}=\frac{x_{i}}{\sqrt{\operatorname{det} x_{i}}}$ and note that $w\left(z_{1}, \ldots, z_{m}\right)=w\left(x_{1}, \ldots, x_{m}\right)$.

For Conjecture 2 we take $y_{i}=x_{i}-I$. Then we can open the brackets in

$$
w\left(1+y_{1}, 1+y_{2}, \ldots, 1+y_{m}\right)=1+f\left(y_{1}, \ldots, y_{m}\right)+g\left(y_{1}, \ldots, y_{m}\right),
$$


where $f$ is a homogeneous Lie polynomial of degree $d$, and $g$ is the sum of terms of degree greater than $d$. Therefore it is interesting to investigate the possible images of Lie polynomials, whether it is possible that the image of $l$ does not contain nilpotent matrices. Unfortunately we saw such an example (1) although its degree must be at least 5 by Spenko [ $\underline{\underline{S}}$, Lemma 7.4]. More general questions about surjectivity of word maps in groups and polynomials in algebras are considered in BeKP.

Remark 4. Our next theorem describes the situation in which the trace vanishing polynomial does not take on nonzero nilpotent values. It implies that any nontrivial word map $w$ evaluated on $\mathrm{PSL}_{2}$ is not surjective iff its projection to $\mathrm{sl}_{2}$ given by $\mathrm{sl}_{2}: x \mapsto x-\frac{1}{2} \operatorname{tr} x$ is a multiple of any prime divisor of $\operatorname{det}(\pi(w))$. This might help in answering Conjecture 1 .

Theorem 4. Let $f\left(x_{1}, \ldots, x_{m}\right)$ be a trace vanishing polynomial, evaluated on $M_{n}(K[\xi])$. Let $\bar{f}=f\left(y_{1}, \ldots, y_{m}\right)$. Then $f$ takes on no nonzero nilpotent values on any integral domain containing $K$, iff each prime divisor $d$ of $\operatorname{det}(\bar{f})$ also divides each entry of $\bar{f}$.

Proof. $(\Rightarrow)$ If some prime divisor $d$ of $\operatorname{det}(\bar{f})$ does not divides $\bar{f}$, then $\bar{f}$ does not specialize to 0 modulo $d$. Therefore we have a nonzero matrix in the image of $f$ which has determinant zero and also trace zero, and thus is nilpotent, a contradiction.

$(\Leftarrow)$ Assume that $f$ takes on a nonzero nilpotent value over some extension integral domain of $K$. Thus $\operatorname{det} \bar{f}$ goes to 0 under the corresponding specialization of the $\xi_{i, j}^{k}$, so some prime divisor $d$ of $\operatorname{det}(\bar{f})$ goes to 0 , and $\bar{f}$ is not divisible by $d$.

\section{REFERENCES}

[Bak] Bakhturin, Yu. A. Identical relations in Lie algebras. VNU Science Press, Utrecht, 1987.

[Ban] Bandman, T., Surjectivity of certain word maps on $\operatorname{PSL}(2, \mathbb{C})$ and $\operatorname{SL}(2, \mathbb{C})$, arXiv:1407.3447

[BGKP] Bandman, T.; Gordeev, N.; Kunyavskii, B.; Plotkin E. Equations in simple Lie algebras, Journal of Algebra 355 (2012), 67-79.

[BeKP] Belov, A.; Kunyavskii, B.; Plotkin, E. Word equations in simple groups and polynomial equations in simple algebras Vestnik St. Petersburg University: Mathematics Surv.46 (2013), 3-13.

[BeKR] Belov, A.; Karasik, Y.; Rowen, L.H. Computational Aspects of Polynomial Identities, 2nd edition, CRC Press, Taylor Francis, Boca Raton, Fl. (2015).

[BeMR1] Belov, A.; Malev, S.; Rowen, L. The images of non-commutative polynomials evaluated on $2 \times 2$ matrices, Proc. Amer. Math. Soc 140 (2012), 465-478.

[BeMR2] Belov, A.; Malev, S.; Rowen, L. The images of multilinear polynomials evaluated on $3 \times 3$ matrices, submitted to the Proc. Amer. Math. Soc. (2013).

[BeMR3] Belov, A.; Malev, S.; Rowen, L., Power-central polynomials on matrices, submitted to the Journal of Pure and Applied Algebra (2013).

[Bo] Borel, A., On free subgroups of semisimple groups, Enseign. Math. 29 (1983), 151-164.

[BrPS] Brear, M., Procesi, C., Špenko, S., Quasi-identities on matrices and the Cayley-Hamilton polynomial arXiv:1212.4597.

[Ch] Chuang, C.-L. On ranges of polynomials in finite matrix rings, Proceeding of the American Mathematical Society 110 (1990), no. 2, 293-302.

[DPP] De Concini, C., Papi, P., and Procesi, C., The adjoint representation inside the exterior algebra of a simple Lie algebra, arXiv:1311.4338. To appear in Advances in Mathematics

[DR] Drensky, V. and Rashkova, T., Weak polynomial identities for the matrix algebras. (English summary) Comm. Algebra 21 (1993), no. 10, 37793795.

[DK] Drensky, V.; Kasparian, A. A new central polynomial for $3 \times 3$ matrices, Commun. Algebra 13 (1985), 745-752.

[Go] Gordienko, A. S., Lecture notes on polynomial identities, preprint (2015)

[G] Gordeev, N. On Engel words on simple algebraic groups., J. Algebra 425 (2015), 215-244. 
[I] Itoh, M., Exterior Algebras and Amitsur-Levitzki type theorems, arXiv:1404.1980

[Ku1] Kulyamin, V. Images of graded polynomials in matrix rings over finite group algebras Russ. Math. Surv.55 (2000), 345-346.

[Ku2] Kulyamin, V. On images of polynomials in finite matrix rings, Thes. Cand. Phys.-Math. Sci., Moscow Lomonosov state University Moscow (2000).

[L] Larsen, M. Word maps have large image. Israel J. Math. 139 (2004), 149-156.

$[\mathrm{M}]$ Malev, S. The images of non-commutative polynomials evaluated on $2 \times 2$ matrices over an arbitrary field. J. Algebra Appl. 13 (2014), no. 6.

[P1] Procesi, C. The invariant theory of $n \times n$ matrices, Advances in Math. 19 (1976), 306-381.

[P2] Procesi, C. On the theorem of Amitsur-Levitzki Israel J. Math. (2014).

[Ra] Razmyslov, Yu. P., Identities of Algebras and Their Representations, Nauka, Moscow (1989), AMS Translations of mathematical monographs 138 (1994).

[Ros] Rosset, S., A new proof of the Amitsur-Levitski identity, Israel J. Math. 23 (1976), no. 2, $187-188$.

[Row] Rowen, L. Polynomial identities in ring theory, Academic press, New York (1980).

$[\check{S}]$ Špenko, S. On the image of a noncommutative polynomial, Journal of Algebra 377 (2013), 298-311.

[Ze] Zelmanov, E. Infinite algebras and pro-p-groups, Progress in Mathematics 248 (2005), 403413.

[Zu] Zubkov, A. Non-abelian pro-p-groups cannot be represented by $2 \times 2$ matrices, Siberian Math. J. 2 (1987), 742-747.

Department of Mathematics, Bar Ilan University, Ramat Gan, Israel

E-mail address: beloval@math.biu.ac.il

Einstein Institute of Mathematics, Hebrew University of Jerusalem, Jerusalem, IsRAEL

E-mail address: malevs@math.huji.ac.il

Department of Mathematics, Bar Ilan University, Ramat Gan, Israel

E-mail address: rowen@math.biu.ac.il 